\title{
THE MASSES OF THE NEUTRON STARS IN M15C
}

\author{
W.T.S. DEICH \\ Netherlands Foundation for Research in Astronomy \\ Postbus 2, 7990 AA Dwingeloo, The Netherlands \\ AND \\ S.R. KULKARNI \\ California Institute of Technology
}

\begin{abstract}
Several years of timing the pulsar in the binary neutron star system M15C have yielded the masses of both stars: the total mass is $M_{\mathrm{T}}=$ 2.7121(6) $\mathrm{M}_{\odot}$; the companion mass is $m_{\mathrm{c}}=1.36(4) \mathrm{M}_{\odot}$; and the pulsar mass is $m_{\mathrm{p}}=1.35(4) \mathrm{M}_{\odot}$. We argue that this system is not likely to have formed through accretion-induced collapse (AIC), and that the standard model also has problems in explaining the formation.
\end{abstract}

\section{Introduction}

Pulsars were discovered in M15 in 1988, as part of an extensive program for searching for millisecond pulsars in globular clusters (Wolszczan et al. 1989; Anderson et al. 1990). There are now eight known pulsars in M15 (Wolszczan et al. 1989; Anderson et al. 1990; Anderson 1993; Middleditch, private communication). Fig. 1 shows the locations of the pulsars in the cluster. The binary pulsar M15C is well outside the core of the cluster, from which it was probably ejected as a result of the collision that formed the system (Phinney \& Sigurdsson 1991).

We report here the results of timing the binary pulsar PSR $2127+11 \mathrm{C}$ (M15C). We have measured the masses of the pulsar and its neutron star companion: both are close to $1.35 \mathrm{M}_{\odot}$, which is inconsistent with the hypothesis that most or even all of the pulsars in globular clusters were formed through accretion-induced collapse (AIC) of massive white dwarfs (Grindlay \& Bailyn 1988; Bailyn \& Grindlay 1990). 


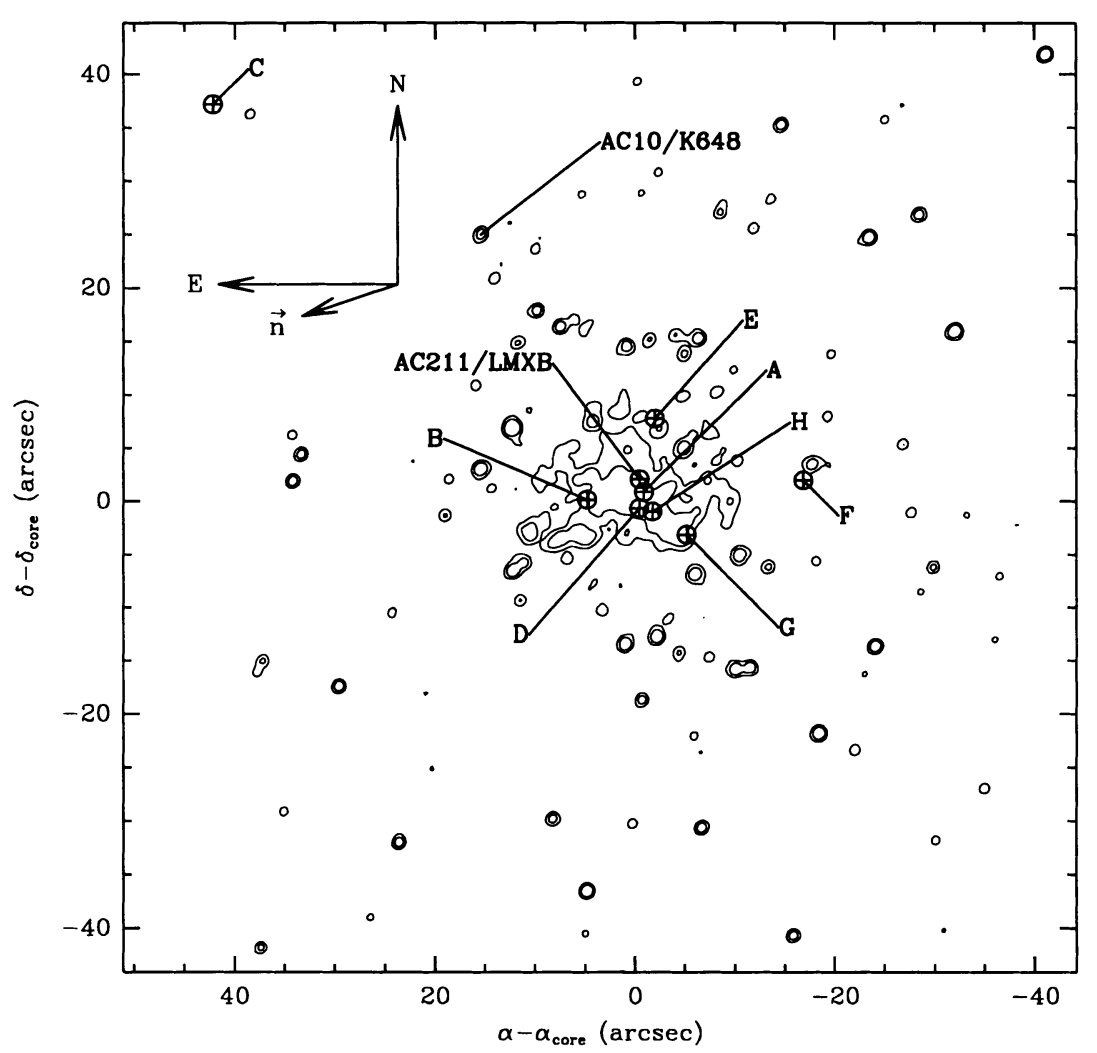

Figure 1. The pulsars superimposed on a VLA image of M15. From Prince et al. (1991).

\section{Observations}

All of the M15 observations were carried out with the Arecibo Observatory's 305-m radio telescope, using dual circularly-polarized line feeds. The center frequencies were either $\nu_{0} \sim 430 \mathrm{MHz}$ or $\nu_{0} \sim 1400 \mathrm{MHz}$ (only PSR 2127+11A is bright enough to obtain useful timing data at $1400 \mathrm{MHz}$ ), using bandwidths $B_{430}=10 \mathrm{MHz}$ and $B_{1400}=40 \mathrm{MHz}$.

The Observatory's three-level, $40 \mathrm{MHz}$ autocorrelation spectrometer was used in two modes. In the first mode ("XCORCHN"), it was divided into 2 polarizations $\times$ four subcorrelators of 32 lags each, for an effective spectral resolution of 2 polarizations $\times 128$ channels across the bandpass. In each subcorrelator, a 32-lag autocorrelation function (ACF's) was formed every $506.625 \mu \mathrm{s} .{ }^{1}$ The correlator data were dumped to tape for off-line analysis,

\footnotetext{
${ }^{1}$ In practice, the autocorrelator samples all lags in $500 \mu \mathrm{s}$, but suffers dead time while it is occupied dumping a block of ACF's to the computer that writes the data to tape. The mean time between lags is precisely $506.625 \mu \mathrm{s}$.
} 
TABLE 1. PSR 2127+11C Timing Parameters.

\begin{tabular}{lcrl}
\hline Pulse Period & $P$ & $30.5292951283(3)$ & $(\mathrm{ms})$ \\
Period Derivative & $\dot{P}$ & $4.9818(80)$ & $10^{-18} \mathrm{~s} \mathrm{~s}^{-1}$ \\
Dispersion Measure & $D M$ & $67.132(1)$ & $\mathrm{cm}^{-3} \mathrm{pc}$ \\
Right Ascension & $\alpha$ & $21^{h} 30^{m} 1^{s} .20548(36)$ & $\mathrm{J} 2000$ coordinates \\
Declination & $\delta$ & $12^{\circ} 10^{\prime} 38^{\prime \prime} .1926(83)$ & $\mathrm{J} 2000$ coordinates \\
Orbital Period & $P_{\mathrm{b}}$ & $28968.36974(2)$ & $\mathrm{s}$ \\
Projected Semi-Major Axis & $a_{1} \sin i$ & $2.51857(21)$ & $\mathrm{ls}$ \\
Eccentricity & $e$ & $0.681388(5)$ & \\
Epoch of Periastron & $T_{0}$ & 2447633.02 & $\mathrm{JD}$ \\
Longitude of Periastron & $\omega_{0}$ & $316^{\circ} .3629$ & \\
Apsidal Motion & $\dot{\omega}$ & 4.4636 & $\mathrm{deg} \mathrm{y}^{-1}$ \\
Einstein Delay & $\gamma$ & 0.00467 & $\mathrm{~ms}$ \\
Orbital Period Decay & $\dot{P}_{\mathrm{b}}$ & -3.937 & $10^{-12} \mathrm{~s} \mathrm{~s}^{-1}$ \\
Total Mass & $M$ & $2.7121(6)$ & $\mathrm{M}_{\odot}$ \\
Companion Mass & $M_{C}$ & $1.363(40)$ & $\mathrm{M}_{\odot}$ \\
\hline
\end{tabular}

allowing the same data set to be used for simultaneous observations of all the pulsars in the beam.

In the second mode ("XCORHI"), 128-lag ACF's of the input voltage were folded synchronously with a Doppler-corrected pulsar period, using 256 pulse phase bins. This was only done for observations of PSR $2127+11 \mathrm{~A}$, yielding an effective time resolution of $\sim 0.43 \mathrm{~ms}$. The integration time was 5 minutes for all of the XCORHI observations.

The observations reported here span the interval 21 February 1988 to 7 November 1993.

\section{The masses of PSR $2127+11 C$ and its companion}

PSR2127+11C, which is much more distant from the core than any of the other seven known pulsars (Fig. 1), is a binary pulsar with a neutron star companion (Anderson 1992). Long-term timing has yielded a measurement of the orbital period decay and the masses of the pulsar and its companion. This is the first accurate mass measurement of a pulsar in a globular cluster, and the fourth accurate binary neutron star mass measurement.

All of the PSR $2127+11 \mathrm{C}$ pulse time of arrival (TOA) measurements are derived from observations at $430 \mathrm{MHz}$. The modest amounts of data available at $1400 \mathrm{MHz}$ did not yield satisfactory TOA's, due to the steep spectral index of this pulsar. Timing from Arecibo continues with monthly 
TABLE 2. PSR 2127+11C Timing Solution Comparison. A comparison of the timing solution to the $1.5 \mathrm{y}$ of data used in Anderson et al. (1992) with the present timing solution.

\begin{tabular}{ll}
\hline Parameter, $X$ & $\Delta(X) / \sigma(X)$ \\
\hline Pulse Period, $P$ & 0.0 \\
Period Derivative, $\dot{P}$ & 0.4 \\
Dispersion Measure, $D M$ & 0.04 \\
Right Ascension, $\alpha$ & 0.2 \\
Declination, $\delta$ & 0.7 \\
Orbital Period, $P_{b}$ & 0.1 \\
Projected Semi-Major Axis, $a_{1} \sin i$ & 0.2 \\
Eccentricity, $e$ & 0.1 \\
Longitude of Periastron, $\omega_{0}$ & 0.1 \\
Total Mass, $M$ & 0.02 \\
Companion Mass, $M_{C}$ & 0.2 \\
\hline
\end{tabular}

observations of M15 at $430 \mathrm{MHz}$. The dispersion was estimated by Anderson (1992) from pulse arrival times across the $10-\mathrm{MHz}$ receiver bandwidth. The timing parameters are presented in Table 1.

We have compared our results with those from the last published timing solution (Anderson 1992), and find good agreement in all parameters. Table 2 compares the fitted parameters from Anderson with the same parameters from our data. The first column gives the parameter, and the second column gives the difference between the Anderson results and ours in units of the uncertainty reported by Anderson. Note that the timing solution reported here includes most of the same data used by Anderson, and that Anderson's uncertainties are conservative, being the formal $3 \sigma$ errors. Thus one should not wonder that for all parameters $X, \Delta(X) / \sigma(X)<1$.

On secular time scales, the apparent period of the pulsar varies due to acceleration of the pulsar and centrifugal contributions. Particularly for pulsars in clusters, the observed parameters can be dominated by external accelerations; for example, PSR 2127+11A has a negative period derivative, which is due to acceleration in the cluster potential. We therefore consider these influences on PSR 2127+11C:

$$
\frac{\dot{P}}{P}=\frac{\dot{P}_{0}}{P_{0}}+\frac{\mathbf{a n}}{c}+\frac{V_{\perp}^{2}}{c D}
$$

where $P_{0}$ is the intrinsic period, a is the pulsar acceleration due to sources outside the binary system, $\mathbf{n}$ is a unit vector from the Earth to the pul- 
sar, $c$ is the speed of light, and $D$ is the pulsar's distance. The observed value for PSR $2127+11 \mathrm{C}$ is $\dot{P} / P=1.610^{-16} \mathrm{~s}^{-1}$. The second term includes accelerations due to passing stars, the cluster potential, and the Galaxy. The mean cluster potential dominates the term, which Phinney (1992) has estimated to be $0.610^{-17} \mathrm{~s}^{-1}$. The third term is an order of magnitude smaller: $V_{\perp}=474 \frac{\mu_{\text {tot }}}{10 \mathrm{masy}^{-1}} \frac{D}{10 \mathrm{kpc}} \mathrm{km} \mathrm{s}^{-1}$, where $\mu_{\mathrm{tot}}$ is the proper motion of the pulsar, and $V_{\perp}^{2} / c D=2.510^{-18} \mathrm{~s}^{-1}$.

Therefore the observed period and period derivatives are a good measure of the intrinsic values, and we conclude that the masses of PSR $2127+11 \mathrm{C}$ and its companion are $1.350(40) \mathrm{M}_{\odot}$ and $1.363(40) \mathrm{M}_{\odot}$, respectively.

\section{Discussion}

Although the binary systems M15C and PSR B1913+16 are superficially similar, with similar orbital period, spin period, and eccentricity, they have very different origins. PSR B1913+16 is a classic product of a high-mass $\mathrm{X}$-ray binary, in which the progenitors were two massive stars in a binary system (Flannery \& Van den Heuvel 1975; Burrows \& Woosley 1986).

In contrast, the $\mathrm{M} 15 \mathrm{C}$ progenitor stars came from different systems and formed a binary after the initial supernova. This can be seen from two separate lines of reasoning: first, the characteristic age of PSR 2127+11C is $\tau_{c}=P / 2 \dot{P}=208 \mathrm{My}$, yet there could not have been any massive stars remaining in the cluster as recently as $200 \mathrm{My}$ ago. Thus PSR $2127+11 \mathrm{C}$ was not spun up by accretion from its present companion.

Second, had the progenitors of the pulsars been in a single binary system, the second explosion would have given the system a large enough kick to escape the cluster. The orbit would have been circular just before the second explosion, and the mass of the helium core that exploded must have been in the range $4-6 \mathrm{M}_{\odot}$ (to produce a companion mass $M_{C}=1.33 \mathrm{M}_{\odot}$; see Burrows \& Woosley 1986). To produce a bound final system with $M_{T}=2.71 \mathrm{M}_{\odot}$ and eccentricity $e=0.68$ would require an asymmetric explosion whose minimum recoil speed is (Burrows \& Woosley 1986; Flannery \& Van den Heuvel 1975)

$$
V_{\min }=710\left(r^{0.5}-(1+e)^{0.5}\right) \mathrm{km} \mathrm{s}^{-1}=80-250 \mathrm{~km} \mathrm{~s}^{-1}
$$

where $r$ is the ratio of the post-explosion to pre-explosion total system mass and $e$ the post-explosion eccentricity.

In the "standard model" for millisecond pulsar production (see for example Bhattacharya \& Van den Heuvel 1991) neutron stars in globular clusters were born from massive stars with $M \geq 6-8 \mathrm{M}_{\odot}$. Most were ejected in the explosion, but some fraction of the retained neutron stars underwent tidal capture and produced low-mass X-ray binaries (LMXBs), which 
evolved to produce the "recycled" spun-up pulsars. The most serious difficulty with the standard model is the so-called birth rate problem, namely that the LMXB lifetime would have to be very short, $\sim 10^{7} \mathrm{yr}$, in order to produce the inferred number of millisecond pulsars.

The competing AIC scenario starts with an accreting binary white-dwarf system. When the accreting white dwarf exceeds the Chandrasekhar limit it collapses directly to a millisecond-period neutron star. A series of papers (e.g., Nomoto 1987; Nomoto \& Kondo 1991) has shown that carbon-oxygen white dwarfs can readily produce neutron stars via AIC. The AIC model has the virtue of avoiding the birth rate problem entirely.

Nonetheless, the masses of PSR 2127+11C and its companion do not fit easily into the AIC scenario. When a white dwarf collapses to a neutron star, it loses $10-15 \%$ of its gravitational binding energy in neutrinos, and $\Delta M \leq 0.02 \mathrm{M}_{\odot}$ in the explosion. The final mass will be in the range 1.23$1.3 \mathrm{M}_{\odot}$. The new neutron star must then accrete about $0.1 \mathrm{M}_{\odot}$ to reach the observed present mass of PSR $2127+11 \mathrm{C}=1.35 \mathrm{M}_{\odot}$. (The companion is not lost, but the orbit grows and becomes eccentric with $e \sim 0.1$.) Yet accreting $0.1 \mathrm{M}_{\odot}$ should lead to a spin period an order of magnitude smaller than observed, and (by comparison with other spun-up pulsars) its magnetic field should also be an order of magnitude less than the inferred $10^{10}$ Gauss.

If the pulsar's mass is taken to be $1.30 \mathrm{M}_{\odot}(1.5 \sigma$ below the best-fit value), then it could have been produced by AIC without requiring any accretion after forming the neutron star. But in that case, the pulsar's companion would have to be $1.41 \mathrm{M}_{\odot}$, and it must have undergone significant accretion after becoming a neutron star.

In summary, AIC cannot account for both neutron stars in M15C without at least one of the members accreting $\sim 0.1 \mathrm{M}_{\odot}$ after collapse to a neutron star.

The standard model also experiences discomfort explaining M15C. Here the prime difficulty is that $B$ is large. If spin-up occurred via a low-mass companion, one would expect $B$ to be as small as other pulsars that have been spun up this way. On the other hand, if PSR $2127+11 \mathrm{C}$ was simply born spinning slow, then the characteristic age of just $200 \mathrm{My}$ implies a very high birth rate of these pulsars.

Acknowledgements. We are grateful to S.B. Anderson, A. Wolszczan, and T.A. Prince for allowing us to use their first 1.5 yrs of M15 time series data in our analysis. 


\section{References}

Anderson, S.B. et al. 1990, Nat 346, 42

Anderson, S.B., 1993, Ph.D. Thesis, California Institute of Technology

Bailyn, C.D. \& Grindlay, J.E. 1990, ApJ 353, 159

Bhattacharya, D. \& Van den Heuvel, E.P.J. 1991, Phys. Rept. 203, 1

Burrows, A. \& Woosley, S.E. 1986, ApJ 308, 680

Flannery, B.P. \& Van den Heuvel, E.P.J. 1975, A\&A 39, 61

Grindlay, J.E. \& Bailyn, C.D. 1988, Nat 336, 48

Nomoto, K. 1987 in The Origin and Evolution of Neutron Stars, IAU Symp. 125, D.J. Helfand \& J.H. Huang (Eds.), Reidel, p. 281

Nomoto, K. \& Kondo, Y. 1991, ApJ 367, L19

Phinney, E.S. 1992, Phil. Trans. Roy. Soc. Lond. A341, 39

Phinney, E.S. \& Sigurdsson, S. 1991, Nat 349, 220

Prince, T.A. et al. 1991, ApJ 374, L41

Wolszczan, A. et al. 1989, Nat 337, 531

\section{Discussion}

J. van Paradijs: Your rejection of the standard evolutionary scenario (which succesfully can explain PSR 1913+16) in the case of M15C depends on the assumption, that the helium star that produced the $1.35 \mathrm{M}_{\odot}$ neutron star, had a mass in the range $4-6 \mathrm{M}_{\odot}$. However, the work of Habets (1986, A\&A 167,61 ) has shown that helium stars with masses as low as $2.2 \mathrm{M}_{\odot}$ may form a neutron star; therefore the system may remain bound as the neutron star is formed. 